\title{
MODELING AND SIMULATION OF PATIENT ADMISSION SERVICES IN A MULTI-SPECIALTY OUTPATIENT CLINIC
}

\author{
Bruno Mocarzel \\ David Shelton \\ Berkcan Uyan \\ Eduardo Pérez \\ Jesus A. Jimenez \\ Ingram School of Engineering \\ Texas State University \\ 601 University Drive \\ San Marcos, TX 78666, USA
}

\author{
Lenore DePagter \\ Live Oak \\ Health Partners \\ 1340 Wonder World Drive \\ San Marcos, TX 78666, USA
}

\begin{abstract}
Tactical planning of resources in healthcare clinics concerns elective patient admission planning and the intermediate term allocation of resource capacities. Its main objectives are to achieve equitable access for patients, to serve the strategically agreed number of patients, and to use resources efficiently. In this paper, we describe a simulation model for an outpatient healthcare clinic facing multiple issues related to patient admission and resource workflow. The main problems identified at the clinic are: 1) phones are not answered promptly and 2) patients experience long wait time to check in and check out. The simulation model focuses on the front desk operations. We investigate different resource allocation policies and report on computational results based on a real clinic, historical data, and both patient and management performance measures.
\end{abstract}

\section{INTRODUCTION}

Long wait times are the major reason for patient service dissatisfaction in healthcare outpatient clinics. A typical patient usually is required to go through a sequence of activities before seeing a physician and most of these activities are performed at the clinic's front desk. For example, some clinics require patients to call in advance to schedule appointments with the physicians. In addition, at the time of the appointment, patients are required to check-in, fill out the requested documentation, and visit with a nurse prior to seeing the physician. Furthermore, patients return to the clinic's front desk after seeing their physician in order to complete the check-out process. Patients expect short waiting times resulting from these activities; otherwise, there will be patient dissatisfaction and inadequate utilization of resources that will impact the clinic's quality of service.

The increase in healthcare costs on local and national stages has propelled the need to reduce costs and improve the efficiency in outpatient services. Over the years, topics related to reducing patient waiting times have received a lot of attention amongst researchers and practitioners. Most of the research done has a focus in improving the clinic scheduling system, but these studies exclude the details regarding the clinic's front desk patient admission processes. More specifically, the clinic is modeled as single-stage servers without processes such as patient calls management, patient check-in and check-out, and documentation. Since physicians are expensive resources and are available for limited time periods, it is criti cal that the services provided at the clinic's front desk should be efficiently conducted and do not limit the capacity of physicians. 
In this paper, a discrete-event simulation is developed to model the patient admission processes occurring at a multi-specialty outpatient clinic front desk. The simulations of the clinic's front desk operations in healthcare is somewhat novel. Our simulation model captures the complexities and interactions occurring at the front desk, which are difficult to capture using analytical techniques. The study focuses on the patient admission processes and assumes the a reliable scheduling appointment system is already in place. The objective of the study is to improve the current operations at the front desk of the clinic to reduce patient waiting times for service, patient waiting time for check-in and check-out, and reduce the number of phone calls unanswered by the front desk staff members at the clinic.

The rest of the paper is organized as follows. In Section 2 we review closely related work. We derive our simulation model and provide a description of the data collection process in Section 3. We report on a computational study in Section 4 and end the paper with some concluding remarks and recommendations for the operation of the clinic front desk in Section 5.

\section{RELATED WORK}

Health care providers are constantly dealing with pressure to reduce costs and improve patient quality of service. Patient waiting time is one of the few tangible service quality elements. Discrete-event simulation has been adopted in multiple healthcare settings to study patient management services; see Jun et al. (1999), Cayirli and Veral (2003), and Gupta and Denton (2008). This technique can be used to forecast the impact of system changes and to investigate the relationship between variables in the system.

For instance, Walter (1973) develop a discrete event simulation model of a hospital radiology department to predict the effects of scheduling policies on the efficiency of the appointment system. The author discuss the performance of the system in terms of the average patient queuing time and doctor idle time during the day. Vanden-Bosch and Dietz (2000) propose a combination of simulation, heuristics, and approximate solutions to reduce a combination of patients' expected waiting times and doctor's overtime. LaGanga and Lawrence (2007) carry out a computer simulation study to estimate providers' overtime and patient waiting times. Their model represents a single provider with deterministic service times and a target overbooking level. They conclude that overbooking can lead to greater throughput without significantly higher waiting times. Pérez et al. (2010) use simulation to model patient service management in nuclear medicine clinics while considering both patient and manager perspectives. Their results provide insights regarding resource allocation policies and patient admissions schedules.

Only few simulation studies investigate clinics environments where patients need to pass through facilities such as registration, check-in, check-out, etc. Swisher et al. (2001) develop a discrete-event simulation model for decision-making in outpatient services. The authors show that the results are very sensitive to changes in the patient mix, patient scheduling, and staffing levels; which are very clinic dependent. Marshall et al. (2005) discuss the importance of understanding the patient flow when analyzing healthcare clinic operations. Harper and Gamlin (2003) show how visual interactive simulation can be used within a structured environment to address wait list issues and build acceptance of results among managers. Other studies addressing the performance of medical clinics using simulation include: El-Darzi et al. (1998) and Rohleder et al. (2007).

\section{SIMULATION MODEL}

\subsection{Description of the Outpatient Clinic}

The outpatient clinic in this study has multiple issues related to patient admission and workflow. The main problems identified at the clinic are: 1) patient complaints about not been able to reach anyone on the phone to schedule their appointments and 2) patients long waiting times to check-in and check-out at the clinic. The clinic schedules most of their patient appointments by phone and patient walk-ins are very limited. All patients dial the same phone number for setting-up appointments with one of the multiple doctors offering services at the clinic. The clinic has four front desk staff members and one manager that 
provides extra help when needed. All the staff members perform multiple tasks and they rotate their position on different days. Figure 1 shows the location of the four staff members at the clinic.

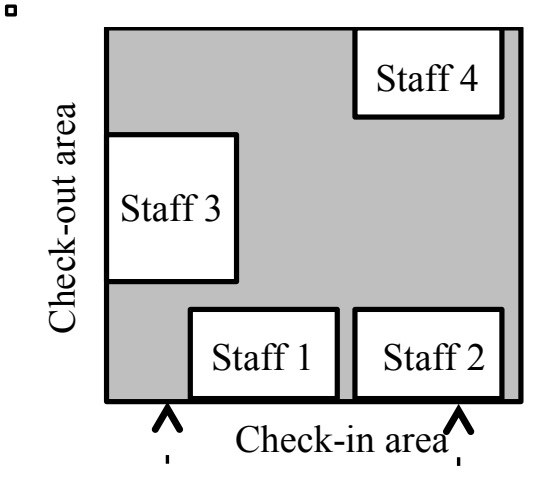

Figure 1: Clinic front desk layout

All four staff members are responsible for answering phones. Staff members 1 and 2 are located at the clinic the main window, and they are primarily responsible for checking-in patients, collecting copays, schedule appointments, scanning/filing documents, medical records, insurance/id cards, verifying benefits, distributing faxes, making copies and at times, if check out window is busy, they help checking-out patients as well. Staff member 3 is positioned in the check-out window. This member of the staff is responsible for patient check-outs, scheduling appointments and follow-ups, collecting copays and deductibles, answering phones, taking messages, making copies, distributing faxes, verifying insurance, and scanning documents and medical records. Staff member 4 sits on the back of the office and is responsible for verifying benefits for all the physicians the day before patient appointments. This staff member is responsible for getting patient paperwork ready and also works on referrals, gets surgery quotes, answers phones, and schedules appointments when the other three staff members are busy.

The clinic has six nurses and six physicians specialized in the following area: orthopedics, general surgeons, and ear nose and throat (ENT doctor). The typical weekly schedule for the physicians is presented in Table 1. Although the focus of this work is on the front desk operations, it is important to understand the correlation between the availability of the doctors and the clinic front desk operations. For example, on those days where most of the physicians are available (i.e. Mondays) a higher volume of patients is expected. In contrast, on those days where few of the doctors are available (i.e. Fridays) a low volume of patients is expected. However, doctors availability do not have a direct impact of the number of calls received at the clinic every day.

Table 1: Weekly Schedule for Physicians

\begin{tabular}{|c|c|c|c|c|c|c|}
\hline Name & Specialty & Monday & Tuesday & Wednesday & Thursday & Friday \\
\hline Doctor 1 & Orthopedics & Shifts 1-2 & - & Shifts $1-2$ & $=$ & Shift 1 \\
\hline Doctor 2 & Orthopedics & Shifts 1-2 & Shifts 1-2 & Shift 1 & Shifts 1-2 & \\
\hline Doctor 3 & ENT & Shifts 1-2 & Shifts 1-2 & Shifts 1-2 & - & Shift 1 \\
\hline Doctor 4 & Surgeon & 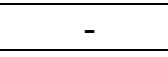 & Shifts 1-2 & - & Shift 1 & - \\
\hline Doctor 5 & Surgeon & Shift 2 & - & Shift 2 & Shift 2 & - \\
\hline Doctor 6 & Surgeon & Shift 1 & & Shift 1 & Shift 1 & Shift 1 \\
\hline
\end{tabular}

Note: Shift 1 is from 9AM-1PM and Shift 2 is from 2PM-5PM

\section{2 $\quad$ Performance Measures}

The performance measures considered in this study involve both patient and management perspectives. The primary performance measures considered in this study were: the waiting time for check-in, the wait- 
ing time for answering phone calls, the number of unanswered calls, the number of patients waiting in queue for check-in, the patient waiting time for check-out, and the front desk staff utilization. These performance measures are used in our simulation model to assess the system performance under different possible operational scenarios at the clinic.

\subsection{Model Abstraction}

The practical setting of the clinic involves four resources (i.e. staff members) and different types of entities such as: patients, phone calls, and documents to be completed. These entities are described in the context of model abstraction and are used to derive the clinic's front desk simulation model.

Staff members are modeled according to their expertise and the type of tasks they can perform as discussed in Section 3.1. In terms of the entities, the model considers two types of patients classified as new or existing. New patients usually require more service time because additional information is required for them to be able to book an appointment and also to check-in at the clinic. Existing patients require less service time for completing those same activities because their information is already in the system. The documents required for providing service to the patients are also considered as entities. Documents are completed by both the patients at the time of check-in and by staff member 4 the day before patient appointments.

A group of flowcharts were developed to understand the activities taking place at the front desk of the clinic and also the interactions between patients and resources. A flowchart was developed for each major process occurring at the front desk of the clinic. Some of the processes considered include: phone calls management, new patient scheduling, existing patient scheduling, patient check-in, and patient check-out. The flowcharts allowed for simplified data collection and also provided the cornerstone for the simulation model development. Figure 2 presents the flowchart for the phone call management process. This flowchart describes the steps followed to service patient calls and some of the tasks include: answering general questions, call transfers, and appointment scheduling and cancelation. Due to space limitations, only one of the flowcharts developed for this project is included in the paper.

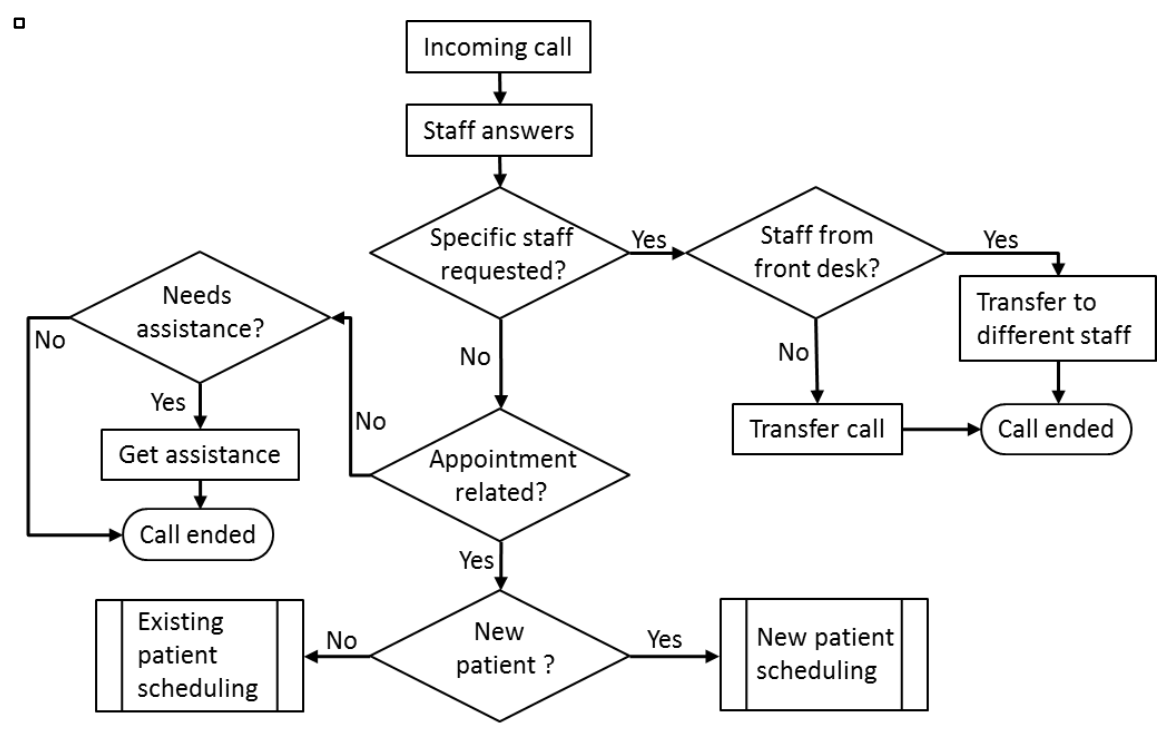

Figure 2: Incoming call flow chart

\subsection{Data}

The data used in this project was collected at the clinic by the first three authors of this paper. A random sampling methodology is used to assure independence among the data collected. The data accounts for 
low and high demand period of times. The flow charts discussed in Section 3.3 aided in the data collection process by identifying those activities important for the operations of the front desk of the clinic. A data collection form was developed using the insight gained developing the flow charts. Figure 3 shows a snapshot of the data collection form and some of the data collected at the clinic, which includes activities related to phone answering.

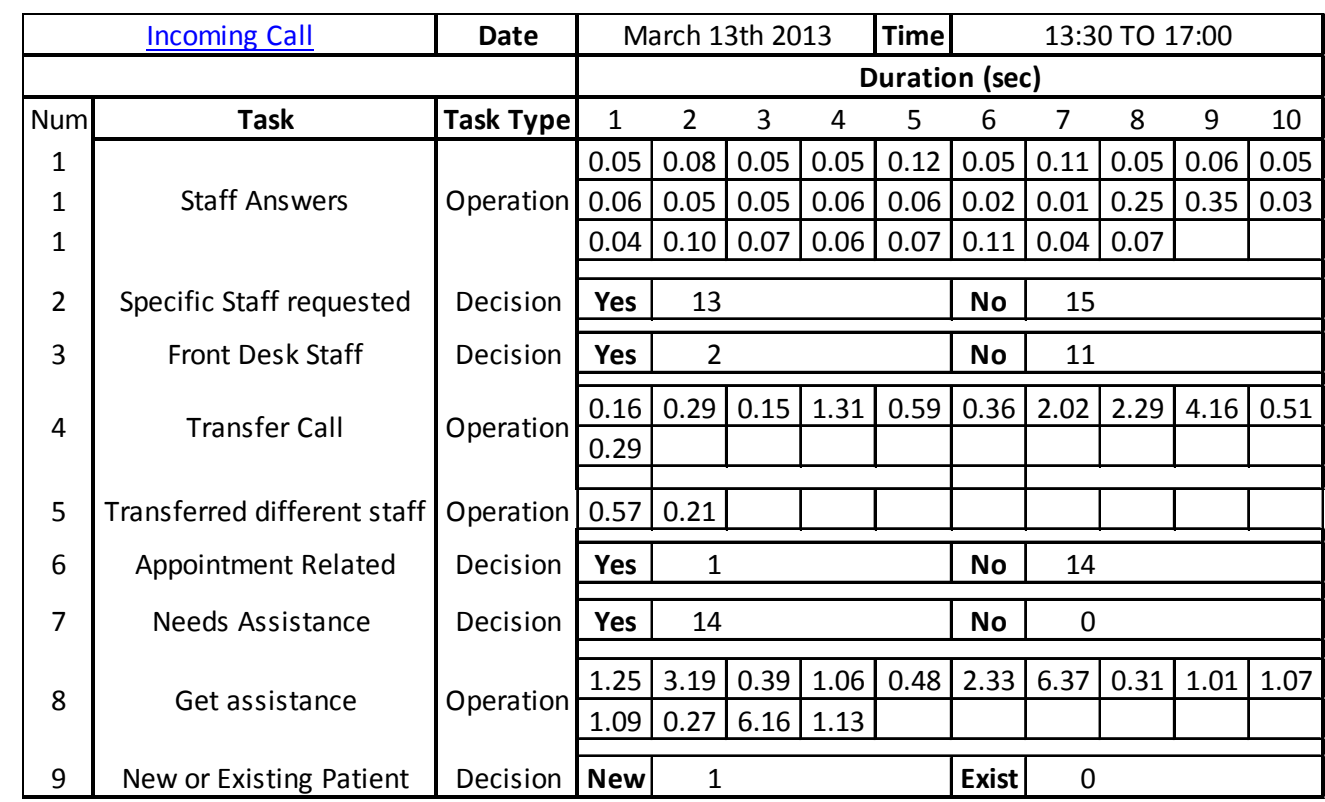

Figure 3: Data collection form

Probability models were developed for each important activity occurring at the front desk of the clinic using the data collected and the Arena Input Analyzer. Figure 4 shows an example of one of the probability distribution fitted for this project. The activity considered in this plot is the time required for transferring calls at the clinic and it follows an exponential distribution.

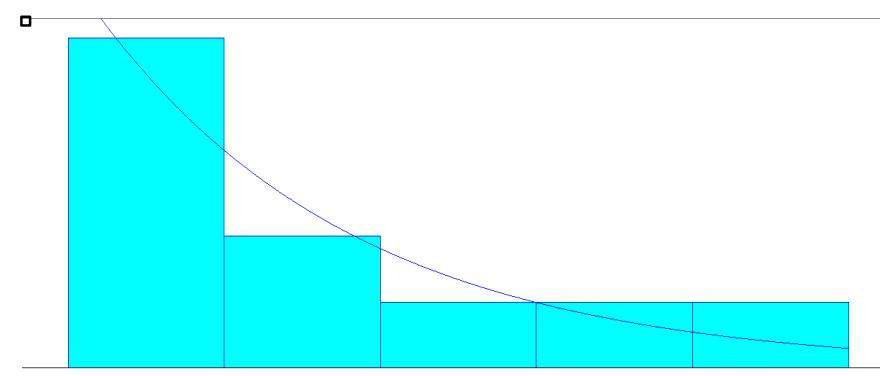

Figure 4: Call transfer times distribution fit

\subsection{Model Implementation, Verification, and Validation}

A discrete-event simulation model for the multi-specialty outpatient clinic front desk was created using the simulation package Arena. Figure 5 provides a snapshot of the model. The simulation model has four major components represented as sub-models. The four major components are: phone calls management, patient check-in, patient check-out, and medical records and documentation management. Each simulation sub-model was created using a flowchart similar to the one discussed in Section 3.3. 
a

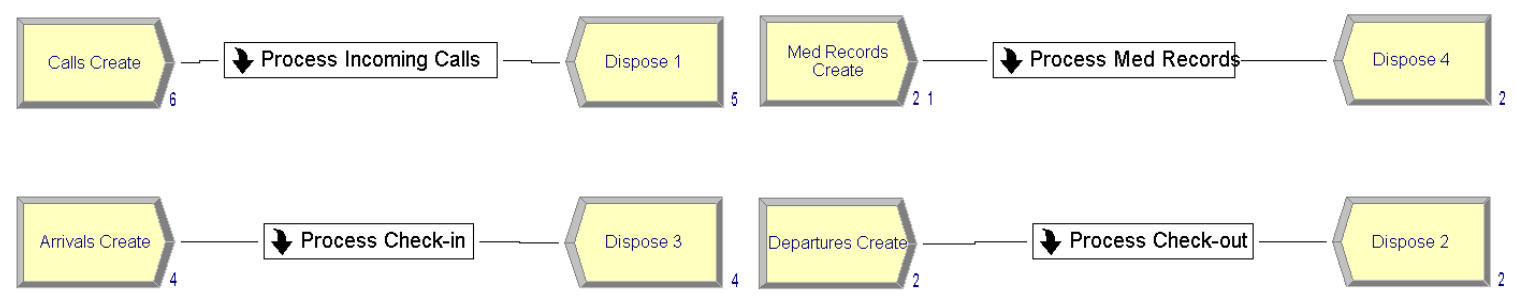

Figure 5: Discrete-event simulation model

A number of techniques were used to verify and validate the simulation model. The animation of the simulation model combined with dynamic statistics provided a general view of the system behavior. Verification was performed by closely examining whether the animation imitates the real system. Validation was done by comparing data obtained at the clinic with the simulated output data for some system performance measures. Figure 6 displays a chart comparing the average of some of the performance measure values obtained from the simulation against the real clinic average values. The results obtained from the simulation indicates that the model provides realistic predictions for the system behavior under various experimental scenarios.

Real clinic Simulation

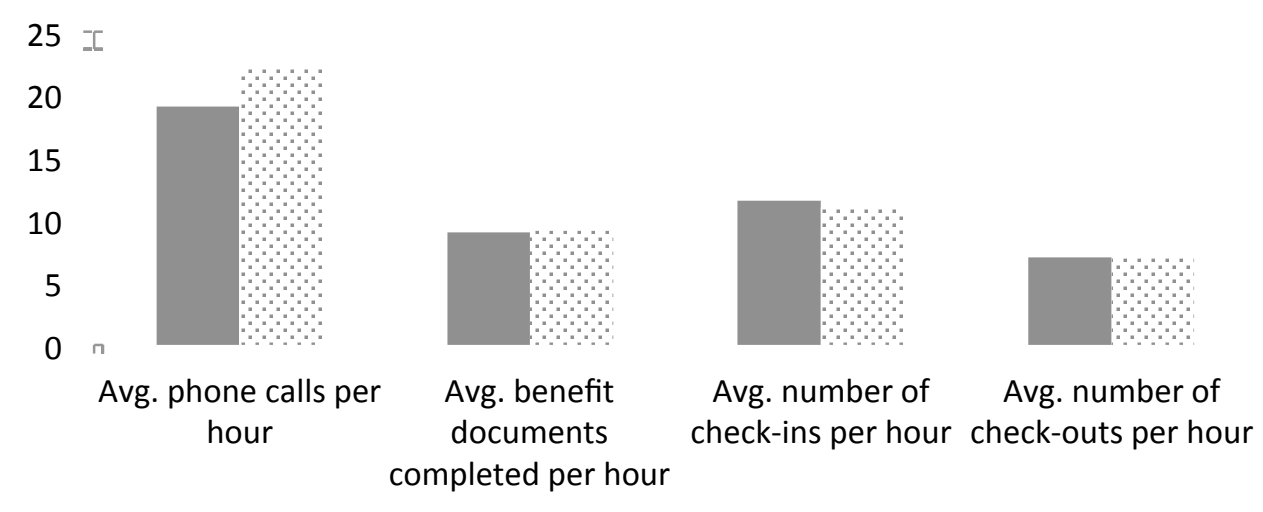

Figure 6: Simulation model performance versus clinic current performance

\section{EXPERIMENTATION}

\subsection{Design of Experiments}

This section provides a discussion and analysis of the statistical experiments performed with the simulation model. A total of 108 experiments were conducted with twenty replications each. The experiments include five main factors (staff capacity, number of calls arriving per hour, number of patients arriving every 15 minutes, percentage of new patients arriving every 15 minutes, and percentage of calls received for scheduling an appointment) and 5 responses (average waiting time for phone answering, average staff member utilization, average check-in waiting time for all types of patients, average number of patients in queue of window 1 and average check-out waiting time for all patients). Table 2 provides the levels for each of the factors in our study.

The factor levels were determined based on preliminary results obtained from a group of pilot experiments. The staff capacity is studied at three different levels. A low level represents the scenario in which 
only three staff members are available at the front desk. This particular scenario was observed multiple times during the study and occurred mostly because extra help from the staff was needed at other sections of the hospital. The normal level represents the clinic current operational conditions with four staff members and the high level is considered to study the performance of the clinic with an extra staff person.

Two factor levels are considered for the phone calls inter arrival times. The low level represents the current phone inter arrival times at the clinic and the high level represents the scenario in which service demand at the clinic is increased. The number of patients arriving every 15 minutes follows the explanation for the phone calls inter arrival times. The low demand factor represents the observed demand at the clinic and the high level factor represents the scenario of the clinic having an increase in their demand. The clinic currently has the capacity for adding extra services and that is why an increment in the patient arrivals can be expected in the near future. The last two factors were studied at three levels with normal representing the current clinic state. The percentage of new patients arriving every 15 minutes has a significant impact in the clinic because new patients usually require more time for check-in.

Table 2: Simulation Experiments

\begin{tabular}{|l|c|c|c|}
\hline \multirow{2}{*}{\multicolumn{1}{|c|}{ Factors }} & \multicolumn{3}{c|}{ Level } \\
\cline { 2 - 4 } & $\operatorname{Low}(L)$ & $\operatorname{Normal}(N)$ & $\operatorname{High}(H)$ \\
\hline Staff Capacity & 3 & 4 & 5 \\
\hline Phone calls inter arrival times (minutes) & $\operatorname{NORM}(2.58,1.17)$ & - & $\operatorname{NORM}(1.58,1.17)$ \\
\hline Number of patients arriving every 15 minutes & $\operatorname{UNIF}(3,5)$ & - & $\mathrm{UNIF}(5,7)$ \\
\hline \% of new patients arriving every 15 minutes & 0.37 & 0.57 & 0.77 \\
\hline \% of calls arriving requesting appointments & 0.55 & 0.75 & 0.95 \\
\hline
\end{tabular}

\subsection{Simulation Results}

The analysis of variance (ANOVA) performed for this simulation study showed that all the factors under consideration are significant. The experimental results are summarized in Figures 7 to 10. These figures have the same format and each presents the results for one performance measures of the simulation study. The lines represent the staff capacity level for the clinic (see legend). The horizontal axis contains all the experimental combinations for the study using the different factor levels presented in Table 2. For instance, the combination HLNL will indicate that the result correspond to the following combination: number of calls per hour (high), number of patients arriving every 15 minutes (low), percentage of new patients arriving every 15 minutes (normal), and percentage of calls arriving requesting appointments (low). A description for the results obtained for each figure is presented next.

\subsubsection{Analysis of patient check-in service}

Figures 7 and 8 depict the average patient check-in waiting time and the number of patients in queue at the clinic for all the scenarios under consideration. The results show that higher check-in waiting times and longer queues are expected when the staff capacity is at their lowest level. The higher waiting times and longer queues occur when capacity levels equals three combined with a high volume of calls per hour, a high number of patients arriving every 15 minutes, and a high percentage of new patients arriving every 15 minutes. The results show that under these conditions having four staff members will reduce the waiting time by about 20 minutes and the queue levels will decrease by 6 patients. Similarly, having a fifth staff member will reduce the waiting check-in times by about 35 minutes and the queue levels will decrease by 9 patients. Figure 7 also shows that higher check-in times are expected for all capacity levels when low volume of calls per hour is combined with a high number of patients arriving every 15 minutes and a high percentage of new patients arriving every 15 minutes. 


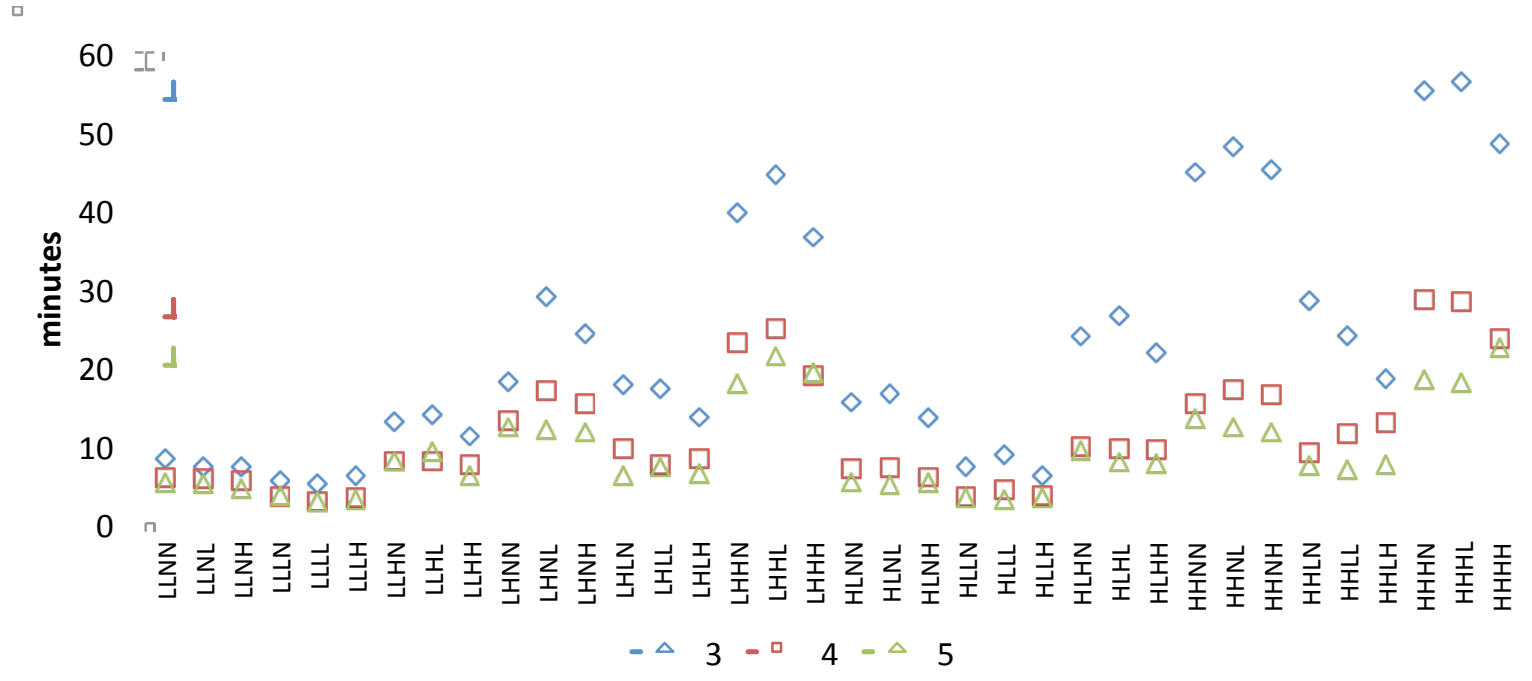

Figure 7: Average patient check-in waiting time
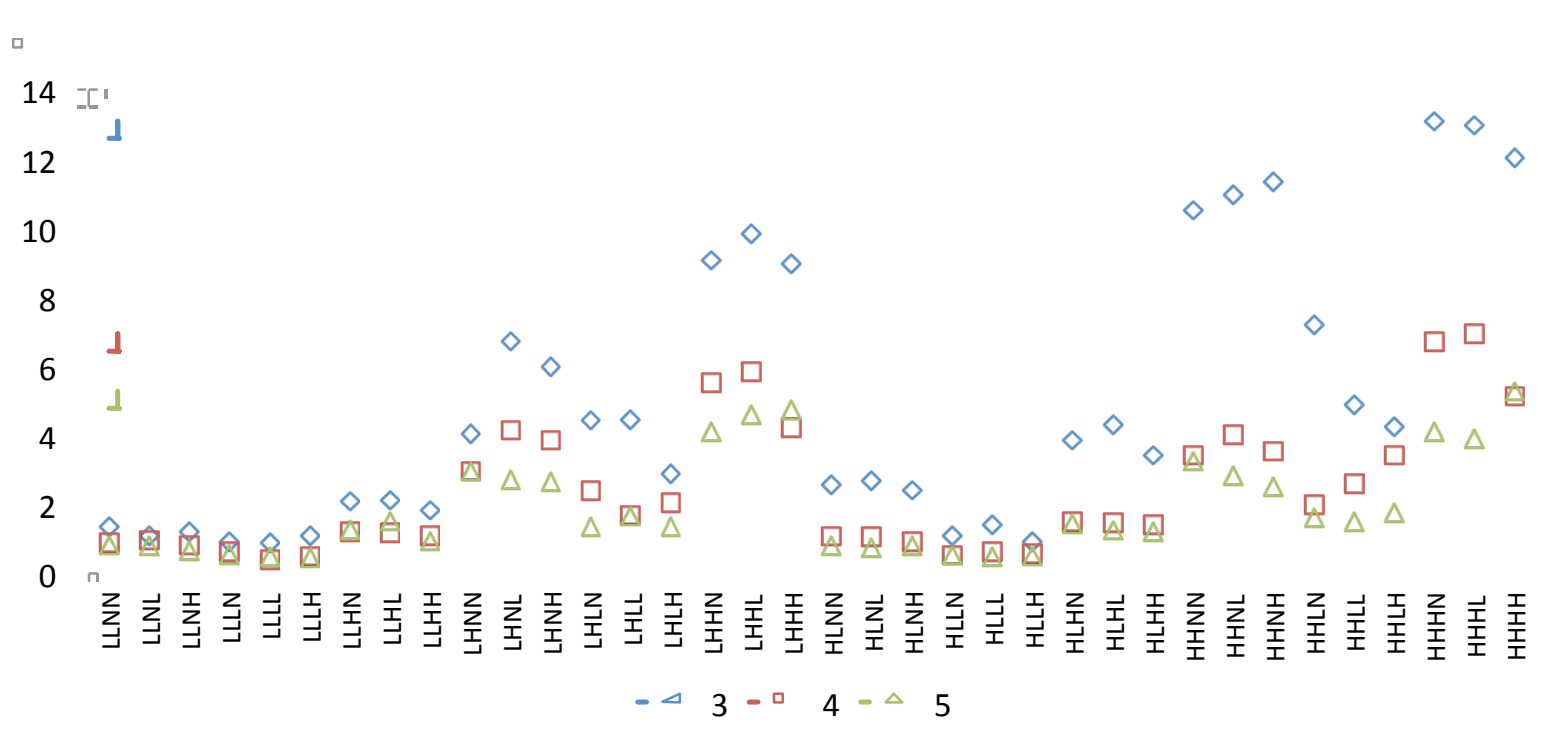

Figure 8: Average number of patients in queue for check-in service

\subsubsection{Analysis of patient phone calls answering service}

The average patient waiting for phone answering is shown in Figure 9 and the average number of phone calls dropped is shown in Figure 10 for all the factor combinations considered in the design of experiments. These simulation results indicate that patients experience significantly higher waiting times when there are three staff members answering the phones. The average waiting times for phone answering decreases when there are four and five staff members at the front desk; however, the difference in this performance metric is nominal at four and five staff members.

The results also show that the system will be able to support a potential increase in the number of calls received at the clinic (i.e. number of calls per hour changes from the low to the high factor level). The best case conditions for higher amounts of phone calls occurs when there are five staff members at the front desk, with average waiting times for phone answering ranging between $0.7-1$ minutes and no dropped phone calls. Similar performance is observed with four staff members, but the number of 
dropped phone calls increases slightly. The worst case occurs when there are three staff members, with the average waiting time ranging between 2-4 minutes and the number of dropped phone calls ranges between 1-8 calls.

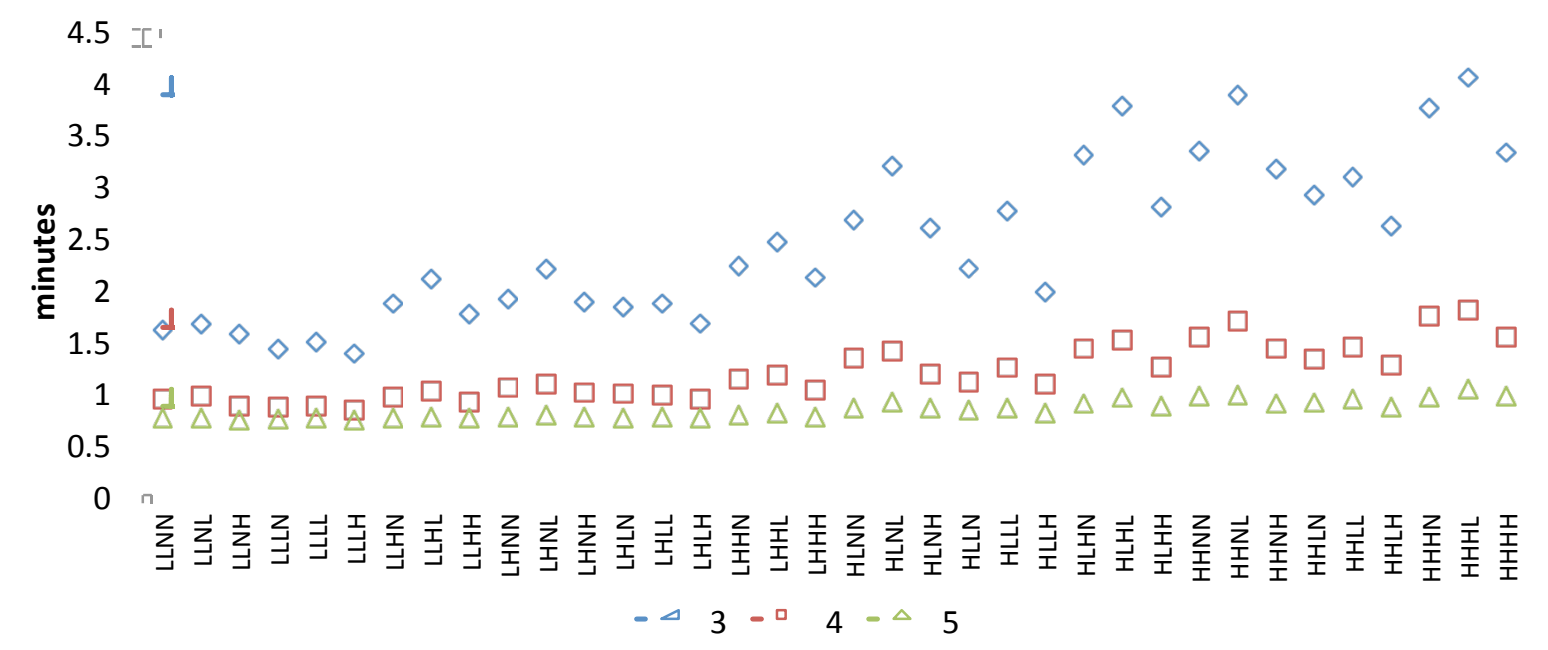

Figure 9: Average patient waiting for phone answering

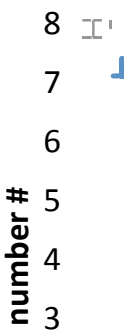

$\downarrow$

6

\# 5

3

2

1

\lrcorner

0

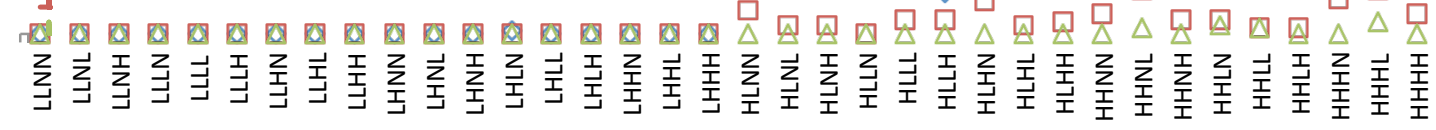

$-\triangle 3-\square \quad 4-\triangle 5$

Figure 10: Average number of calls dropped

\section{CONCLUSIONS AND RECOMMENDATIONS}

In small-size outpatient clinics, several important metrics measure the quality of customer service at patients admissions, such as the waiting time that patients wait while scheduling appointments or while checking-in at the clinic. Management of the clinics controls these quality metrics by providing an adequate number of staff members at the front desk. In this paper, a simulation model was developed in order to analyze the patient admission process, which includes: setting up appointments by phone, insurance verification, and patient check in and check out at the clinic. Factors affecting these services were identified as the number of staff members at the front desk, number of phone calls received, number of patients arrivals, percentage of new patients during arrivals, percentage of phone calls requesting new appointments. A design of experiments was conducted and analyzed in order to evaluate how these factors and the factor interactions impact average waiting time at check-in, average number of patients waiting in 
queue, average waiting time for phone call answering, and average number of dropped phone calls. Several suggestions and implications evolve from this simulation study:

1. There should be a balance in the schedule of new and existing patients throughout the day. Since it takes a longer time to check-in a new patient, having multiple new patients arriving at the same time increases the waiting times at the front desk. Also, the extra time involved in checking-in new patients would be obstacle for answering calls.

2. The use of automated services should allow staff members to be better utilized. For example, an user-friendly automated attendant service (i.e. switchboard) should be implemented for answering incoming calls. In our study, most of the patients calling the front desk needed assistance on nurse-related issues, and therefore the switchboard would direct these calls directly to the nurse's desk.

3. Simulation modeling and analysis enables quantitative decision making for managing health care clinics. In our simulated system study, we observed that there would be no need for an additional staff member at the front desk. In addition, the results can be used as guidelines for deciding when will be appropriate to operate with only three staff members.

Future research work should focus in integrating the clinic's front desk services into the process of scheduling physicians. The objective would be to improve patient satisfaction and quality of service by eliminating bottlenecks and decreasing patients wait time, particularly when there is a significant overlap of physicians at small-size clinics.

\section{ACKNOWLEDGEMENTS}

The authors would like to thank the staff at the Central Texas Medical Center (CTMC) for their assistance and feedback including Yesenia Castillo and Sam Huenergardt. We would like to thank Dr. Tongdan Jin for the support provided in the development of the simulation model abstraction and in the analysis of the results.

\section{REFERENCES}

Cayirli, T. and E. Veral. 2003. "Outpatient scheduling in health care: A review of literature." Production and Operations Management 12(4): 519--549.

El-Darzi, E., C. Vasilakis, T. Chaussalet and P. Millard. 1998. "A simulation modelling approach to evaluating length of stay, occupancy, emptiness and bed blocking in a hospital geriatric department." Health Care Management Science 1(2): 143-149.

Gupta, D. and B. Denton. 2008. "Appointment scheduling in health care: Challenges and opportunities." IIE Transactions 40(9): 800-819.

Harper, P. and H. Gamlin. 2003. "Reduced outpatient waiting times with improved appointment scheduling: a simulation modelling approach." OR Spectrum 25(2): 207-222.

Jun, J. B., S. H. Jacobson and J. R. Swisher. 1999. "Application of discrete-event simulation in health care clinics: a survey." The Journal of the Operational Research Society 50(2): 25.

LaGanga, L. R. and S. R. Lawrence. 2007. "Clinic overbooking to improve patient access and increase provider productivity." Decision Sciences 38(2): 251--276.

Marshall, A., C. Vasilakis and E. El-Darzi. 2005. "Length of stay-based patient flow models: recent developments and future directions." Health Care Management Science 8(3): 213-220.

Pérez, E., L. Ntaimo, C. Bailey and P. McCormack. 2010. "Modeling and simulation of nuclear medicine patient service management in DEVS." Simulation 86(8-9): 481-501.

Rohleder, T. R., D. P. Bischak and L. B. Baskin. 2007. "Modeling patient service centers with simulation and system dynamics." Health Care Management Science 10(1): 1-12. 
Swisher, J. R., S. H. Jacobson, J. B. Jun and O. Balci. 2001. "Modeling and analyzing a physician clinic environment using discrete-event (visual) simulation." Computers and Operations Research 28(2): 105--125.

Vanden-Bosch, P. M. and D. C. Dietz. 2000. "Minimizing expected waiting in a medical appointment system " IIE Transactions 32: 841-848.

Walter, S. 1973. "A comparison of appointment schedules in a hospital radiology department." British journal of preventive \& social medicine 27(3): 160-167.

\section{AUTHOR BIOGRAPHIES}

BRUNO MOCARZEL is an undergraduate student at Texas State University-San Marcos pursuing a degree in Industrial Engineering. He is expected to graduate from Texas State University-San Marcos in December 2013. Bruno has implemented process improvement at Tokyo Electron America, Inc. and the Center for Mathematics Readiness at Texas State University-San Marcos as an undergraduate student.

DAVID SHELTON is an undergraduate student at Texas State University-San Marcos pursuing a degree in Industrial Engineering. He is expected to graduate from Texas State University-San Marcos in December 2013..

BERKCAN UYAN is a recent graduate with a Bachelor of Science degree in Industrial Engineering from Texas State University, Ingram School of Engineering in San Marcos, Texas, USA. He has minors in Applied Mathematics and Business Administration from the same university.

EDUARDO PEREZ is an Assistant Professor at Texas State University, Ingram School of Engineering, San Marcos, Texas, USA. He obtained his Ph.D. in Systems and Industrial Engineering from the Texas A\&M University. His research interests include healthcare systems engineering and analysis, patient and resource scheduling, and optimization techniques . He is a member of INFORMS and IIE. His email address is eduardopr@txstate.edu.

JESUS A. JIMENEZ is an Associate Professor in the Ingram School of Engineering at Texas State University. He received his B.S. and M.S. in Industrial Engineering from The University of Texas at El Paso, and his Ph.D. in Industrial Engineering from Arizona State University. His research interests are in simulation modeling and analysis of manufacturing systems; discrete-event and agent-based simulation; design of simulation experiments; and sustainable lean manufacturing. He is member of INFORMS and IIE. His email address is jesus.jimenez@txstate.edu.

LENORE DEPAGTER is Physician Practice Administrator of Live Oak Health Partners. She obtained a degree of Doctor of Osteopathy from the University of North Texas Health Science Center- Fort Worth and completed a combined residency in Internal Medicine and Pediatrics at Scott \& White Memorial Hospital. She is a current MBA candidate at the University of Texas at Dallas/Southwestern Medical Center. She is an alumni of Texas State University. 